\title{
THE DOUBLE ROMAN DOMATIC NUMBER OF A DIGRAPH
}

\author{
LUTZ VOLKMANN \\ Lehrstuhl II für Mathematik \\ RWTH Aachen University \\ 52056 Aachen, Germany \\ e-mail: volkm@math2.rwth-aachen.de
}

\begin{abstract}
A double Roman dominating function on a digraph $D$ with vertex set $V(D)$ is defined in [G. Hao, X. Chen and L. Volkmann, Double Roman domination in digraphs, Bull. Malays. Math. Sci. Soc. (2017).] as a function $f: V(D) \rightarrow\{0,1,2,3\}$ having the property that if $f(v)=0$, then the vertex $v$ must have at least two in-neighbors assigned 2 under $f$ or one in-neighbor $w$ with $f(w)=3$, and if $f(v)=1$, then the vertex $v$ must have at least one in-neighbor $u$ with $f(u) \geq 2$. A set $\left\{f_{1}, f_{2}, \ldots, f_{d}\right\}$ of distinct double Roman dominating functions on $D$ with the property that $\sum_{i=1}^{d} f_{i}(v) \leq 3$ for each $v \in V(D)$ is called a double Roman dominating family (of functions) on $D$. The maximum number of functions in a double Roman dominating family on $D$ is the double Roman domatic number of $D$, denoted by $d_{d R}(D)$. We initiate the study of the double Roman domatic number, and we present different sharp bounds on $d_{d R}(D)$. In addition, we determine the double Roman domatic number of some classes of digraphs.
\end{abstract}

Keywords: digraph, double Roman domination, double Roman domatic number.

2010 Mathematics Subject Classification: 05C20, 05C40.

\section{REFERENCES}

[1] H. Abdollahzadeh Ahangar, J. Amjadi, M. Atapour, M. Chellali and S.M. Sheikholeslami, Double Roman trees, Ars Combin., to appear.

[2] H. Abdollahzadeh Ahangar, J. Amjadi, M. Chellali, S. Nazari-Moghaddam and S.M. Sheikholeslami, Trees with double Roman domination number twice the domination number plus two, Iran. J. Sci. Technol. Trans. A Sci. (2018), in press. doi:10.1007/s40995-018-0535-7 
[3] H. Abdollahzadeh Ahangar, M. Chellali and S.M. Sheikholeslami, On the double Roman domination in graphs, Discrete Appl. Math. 232 (2017) 1-7. doi:10.1016/j.dam.2017.06.014

[4] R.A. Beeler, T.W. Haynes and S.T. Hedetniemi, Double Roman domination, Discrete Appl. Math. 211 (2016) 23-29. doi:10.1016/j.dam.2016.03.017

[5] G. Hao, X. Chen and L. Volkmann, Double Roman domination in digraphs, Bull. Malays. Math. Sci. Soc. (2017), in press. doi:10.1007/s40840-017-0582-9

[6] T.W. Haynes, S.T. Hedetniemi and P.J. Slater, Fundamentals of Domination in Graphs (Marcel Dekker, Inc., New York, 1998).

[7] N. Jafari Rad and H. Rahbani, Some progress on double Roman domination in graphs, Discuss. Math. Graph Theory, in press. doi:10.7151/dmgt.2069

[8] E.A. Nordhaus and J.W. Gaddum, On complementary graphs, Amer. Math. Monthly 63 (1956) 175-177. doi: $10.2307 / 2306658$

[9] L. Volkmann, The double Roman domatic number of a graph, J. Combin. Math. Combin. Comput. 104 (2018) 205-215.

[10] B. Zelinka, Domatic number and degrees of vertices of a graph, Math. Slovaca 33 (1983) 145-147.

[11] X. Zhang, Z. Li, H. Jiang and Z. Shao, Double Roman domination in trees, Inform. Process. Lett. 134 (2018) 31-34. doi:10.1016/j.ipl.2018.01.004

Received 8 March 2018

Revised 15 June 2018

Accepted 15 June 2018 